\title{
Transarterial chemoembolization combined with radiofrequency ablation in the treatment of hepatocellular carcinomas larger than $5 \mathrm{~cm}$
}

\author{
Yi Yang', Zhuo-Min Lv', Min Yan², Hong-Xin Zhang ${ }^{1, \#, ~ Y o n g ~ L o n g ~}{ }^{2, \#, W e i-L u ~ Z h a n g ~}{ }^{2, \#}$ \\ 'Department of Pain Treatment, Tangdu Hospital of the Fourth Military Medical University, Xi'an 710038, China. \\ ${ }^{2}$ Department of Epidemiology, Ministry of Education Key Lab of Hazard Assessment and Control in Special Operational Environment, \\ the Fourth Military Medical University, Xi'an 710032, China. \\ \#Authors contributed equally to this work.
}

\begin{abstract}
Correspondence to: Dr. Wei-Lu Zhang and Dr. Yong Long, Department of Epidemiology, Ministry of Education Key Lab of Hazard Assessment and Control in Special Operational Environment, the Fourth Military Medical University, No. 169 Changle west road, Xi'an 710032, China. E-mail: zhangweilu@126.com; longyong@fmmu.edu.cn; Hong-Xin Zhang, Department of Pain Treatment, Tangdu Hospital of the Fourth Military Medical University, No. 569 Xinsi Road, Xi'an 710038, China. E-mail: zhhxtdjr@163.com
\end{abstract}

How to cite this article: Yang Y, Lv ZM, Yan M, Zhang HX, Long Y, Zhang WL. Transarterial chemoembolization combined with radiofrequency ablation in the treatment of hepatocellular carcinomas larger than $5 \mathrm{~cm}$. Hepatoma Res 2019;5:13.

http://dx.doi.org/10.20517/2394-5079.2019.04

Received: 8 Jan 2019 First Decision: 18 Feb 2019 Revised: 11 Mar 2019 Accepted: 12 Mar 2019 Published: 26 Apr 2019

Science Editor: Guang-Wen Cao Copy Editor: Cai-Hong Wang Production Editor: Huan-Liang Wu

\section{Abstract}

Aim: This meta-analysis was designed to compare the effectiveness of the combination of transarterial chemoembolization (TACE) and radiofrequency ablation (RFA) vs. that of TACE alone in hepatocellular carcinoma (HCC) tumors larger than $5 \mathrm{~cm}$.

Methods: PUBMED, CNKI, and CBM were searched for all related randomized controlled trials (RCTs) up until October 22, 2018. Eleven studies were identified that compared TACE with RFA vs. TACE alone for HCC treatment. Tumor response rate, the proportion of patients with either complete or partial shrinkage of tumors, and survival rate were the major evaluation indices.

Results: Meta-analysis data revealed that TACE with RFA showed significantly better tumor response rate (risk ratio $(R R)=1.452,95 \%$ confidence interval $(C l): 1.308-1.610, P<0.001)$ and 1-year overall survival rate $(R R=1.412,95 \%$ $\mathrm{Cl}: 1.249-1.596, P<0.001)$ than that of TACE alone treatment.

Conclusion: The data of our study indicates that TACE combined with RFA in the treatment of HCC larger than $5 \mathrm{~cm}$ is an effective comprehensive interventional therapy.

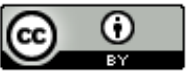

(C) The Author(s) 2019. Open Access This article is licensed under a Creative Commons Attribution 4.0 International License (https://creativecommons.org/licenses/by/4.0/), which permits unrestricted use sharing, adaptation, distribution and reproduction in any medium or format, for any purpose, even commercially, as long as you give appropriate credit to the original author(s) and the source, provide a link to the Creative Commons license, and indicate if changes were made.

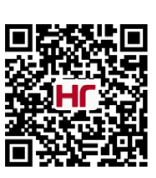


Keywords: Transarterial chemoembolization; radiofrequency ablation; hepatocellular carcinoma; meta-analysis

\section{INTRODUCTION}

Hepatocellular carcinoma (HCC) is one of the most common and malignant tumor in the world, with an annual incidence of over 700,000 patients worldwide ${ }^{[1]}$. As the symptoms of HCC often do not present in the early stages, most patients are in the middle and late stage at the time of diagnosis, among which only $20 \%-30 \%$ of patients have the chance to receive surgical resection or liver transplantation ${ }^{[2]}$. Patients with large tumors that cannot undergo surgical resection or liver transplantation are usually offered comprehensive treatment based on transarterial chemoembolization $(\mathrm{TACE})^{[3,4]}$. However, the long-term outcome of treating HCC with TACE alone is not ideal, due to incomplete tumor necrosis ${ }^{[5,6]}$. Studies have shown that TACE combined with RFA in the treatment of HCC is more efficacious than either TACE or RFA alone ${ }^{[7,8]}$. Nevertheless, some studies have reported contradictory results ${ }^{[9,10]}$. Of note, the sample sizes of these studies are small and the observations need further validation. Additionally, it is unknown whether this combined treatment is more effective than single modality treatment for HCC tumors larger than $5 \mathrm{~cm}$.

Therefore, in order to determine whether TACE plus RFA is more effective in patients with HCC than TACE alone, this current meta-analysis was performed to compare the efficacy of TACE plus RFA with TACE monotherapy. This comparison is expected to provide more convincing evidence for HCC patients having to choose between two methods. In this study, the clinical efficacy of TACE combined with RFA was compared with that of TACE alone in the treatment of HCC larger than $5 \mathrm{~cm}$, to provide evidence to guide clinical practice.

\section{METHODS}

\section{Search methods and quality assessment}

As of October 22, 2018, randomized controlled trials (RCT) comparing the clinical efficacy of TACE with RFA vs. TACE alone in the treatment of HCC was performed using a computerized search on PUBMED, Chinese Journal Full-text Database (CKNI), and CBM. Search terms include "Liver Neoplasms/therapy" [Mesh], "Chemoembolization, Therapeutic" [Mesh], "TACE”, "Radiofrequency ablation”. The literature language is limited to Chinese and English.

Evaluation of literature quality (including literature data extraction and quality scoring) was carried out by the authors. According to the Jadad quality standard, the scoring method is as follows. Whether it is randomly assigned: 2 points is awarded for detailed random allocation, 1 point when it was not specifically described, and 0 point if it was not mentioned. Whether analysis was blinded, 2 points for double-blind, 1 point for blinding without detailed description, o point for open trial. Whether there was a detailed reason for loss of follow-up: 1 point for yes, 0 point for no. High quality research literatures are those that received 3 to 5 points; and low quality literatures are those that received 0 to 2 points.

\section{Inclusion criteria}

Literature reports were eligible for inclusion if: (1) they are domestic or international publications, that compared the clinical efficacy of TACE combined with RFA vs. TACE alone in the treatment of intermediate and advanced staged HCC; (2) they report complete case data; (3) the results of the study include tumor response rate; (4) the maximum diameter of tumor lesions is greater than $5 \mathrm{~cm}$; (5) the clinical study design is consistent with that of a RCT.

\section{Exclusion criteria}

Literature reports were excluded if: (1) they are review articles or case reports, are of poor literature quality as evaluated by the above method, or have no proper controls; (2) they are animal studies; (3) there are 
Table 1. Main characteristics of studies concerning tumor response rate between TACE with RFA vs. TACE alone

\begin{tabular}{|c|c|c|c|c|c|c|c|c|c|}
\hline \multirow{2}{*}{ Ref. } & \multirow{2}{*}{$\begin{array}{l}\text { Year study was } \\
\text { conducted }\end{array}$} & \multirow{2}{*}{ Gender } & \multirow{2}{*}{$\begin{array}{l}\text { Sample } \\
\text { size }\end{array}$} & \multicolumn{2}{|c|}{ TACE } & \multirow{2}{*}{$\begin{array}{c}\text { Tumor } \\
\text { response rate }\end{array}$} & \multicolumn{2}{|c|}{ TACE + RFA } & \multirow{2}{*}{$\begin{array}{c}\text { Tumor } \\
\text { response rate }\end{array}$} \\
\hline & & & & Total & Events & & Total & Events & \\
\hline Dong et al. ${ }^{[11]}$ & $2011-2012$ & Both & 44 & 22 & 6 & 0.272727273 & 22 & 11 & 0.5 \\
\hline Duet al. ${ }^{[12]}$ & $2015-2016$ & Both & 80 & 40 & 14 & 0.35 & 40 & 23 & 0.575 \\
\hline Ge and Zhang ${ }^{[13]}$ & 2008-2009 & Both & 43 & 24 & 12 & 0.5 & 19 & 14 & 0.736842105 \\
\hline Kuang et al. ${ }^{[14]}$ & $2015-2017$ & Both & 87 & 40 & 21 & 0.525 & 47 & 35 & 0.744680851 \\
\hline Li et al. ${ }^{[15]}$ & $2012-2013$ & Both & 80 & 42 & 21 & 0.5 & 38 & 27 & 0.710526316 \\
\hline $\operatorname{Liang}^{[16]}$ & $2006-2008$ & Both & 55 & 24 & 9 & 0.375 & 31 & 25 & 0.806451613 \\
\hline Liu et al. ${ }^{[17]}$ & $2011-2013$ & Both & 128 & 64 & 10 & 0.15625 & 64 & 22 & 0.34375 \\
\hline Shen et $a{ }^{[18]}$ & 2004-2005 & Both & 40 & 19 & 9 & 0.473684211 & 21 & 17 & 0.80952381 \\
\hline Song et al. ${ }^{[19]}$ & $2006-2008$ & Both & 29 & 15 & 4 & 0.266666667 & 14 & 11 & 0.785714286 \\
\hline Zhang et al. ${ }^{[20]}$ & 2012-2014 & Both & 70 & 33 & 6 & 0.181818182 & 37 & 17 & 0.459459459 \\
\hline Yang et al. ${ }^{[21]}$ & $2006-2008$ & Both & 35 & 11 & 6 & 0.545454545 & 24 & 16 & 0.666666667 \\
\hline
\end{tabular}

duplicate reports of similar content by the same author, or if there are too few patients and unclear data; (4) the maximum diameter of tumor lesions is less than $5 \mathrm{~cm}$.

\section{Data acquisition}

The literature and extracted the data were screened independently by authors. After articles were screened by their titles and abstracts, they were filtered by reading the full text. During the screening process, the literature was selected in strict accordance with the set inclusion and exclusion criteria. After the screening was completed, the articles were read again to verify that they meet the requirements.

\section{Statistical methods}

Statistical analysis was performed using Comprehensive Meta Analysis V2. Before the meta-analysis, the heterogeneity $I^{2}$ test of each test result was performed. If the homogeneity of each test included in the study was good $(P>0.05)$, the fixed effect model was used. If heterogeneity existed, the random effect model was used. A funnel chart was used to evaluate the bias risk of the inclusion test, and asymmetric funnel charts suggest that there may be publication bias.

\section{RESULTS}

\section{Literature search results}

Manual search of electronic databases identified a total of 1,487 studies. After checking for duplicates, there were 1,304 remaining. A large number of these studies were excluded based upon our inclusion and exclusion criteria, leaving only 11 articles to be included in the meta-analysis [Figure 1 and Table 1].

\section{Tumor response rate}

There were 11 reports with tumor response rate data comparing TACE with RFA vs. TACE alone. Tumor response rate was measured by the proportion of patients with either complete or partial shrinkage of tumors. Since the heterogeneity test had a $P=0.983$, the fixed-effects model was used. The results showed that the tumor response rate of TACE with RFA in the treatment of HCC was significantly superior to TACE alone [risk ratio $(\mathrm{RR})=1.452$, 95\%CI: 1.308-1.610, $P<0.001, I^{2}=0 \%$ ] [Figure 2].

\section{Six-month survival rate}

Six studies ${ }^{[15,16,18-21]}$ (involving 309 participants) compared the half-year survival of the TACE with RFA group vs. the TACE alone group. The results showed that half-year survival rate was higher in the TACE with RFA group than in the TACE alone group $\left[\mathrm{RR}=1.257,95 \% \mathrm{CI}=1.128-1.401, P<0.001, I^{2}=0 \%\right.$ ] [Figure 3].

\section{One-year survival rate}

Eight studies ${ }^{[1-21]}$ (involving 524 participants) compared the 1-year survival of the TACE with RFA group vs. the TACE alone group. The results showed that 1-year survival rate was higher in the TACE with RFA group compared to the TACE alone group $\left[\mathrm{RR}=1.412,95 \% \mathrm{CI}=1.249-1.596, P<0.001, I^{2}=0 \%\right.$ ] [Figure 4]. 


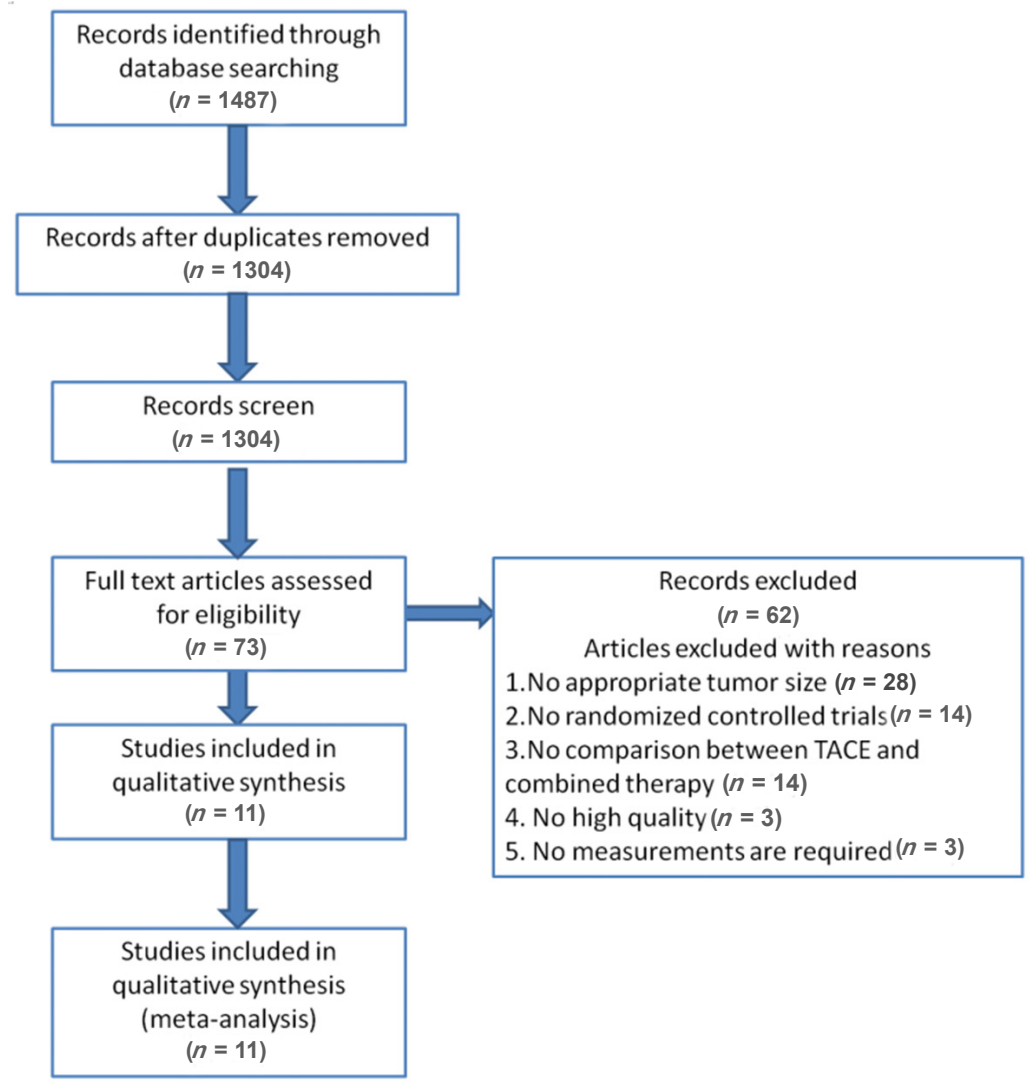

Figure 1. Flow diagram of the detailed selection process of this meta-analysis. A total of $11 \mathrm{RCTs}^{[11-21]}[$ Table 1] were included in the study. There were 691 eligible patients, of whom 357 received TACE with RFA, and 334 received TACE alone. The baseline characteristics of the trials included in the meta-analysis are shown in Table 1. The quality of the included studies was assessed using the Cochrane Collaboration Tool

\section{Eighteen-month survival rate}

Six studies ${ }^{[15,16,18-21]}$ (involving 309 participants) compared eighteen-month survival of the TACE with RFA group vs. the TACE alone group. The results showed that eighteen-month survival rate was higher in the TACE with RFA group than in the TACE alone group [RR $=1.792,95 \% \mathrm{CI}: 1.423-2.256, P<0.001, I^{2}=0 \%$ ] [Figure 5].

\section{Two-year survival rate}

Three studies ${ }^{[14,17,20]}$ (involving 285 participants) compared the 2-year survival rate of the TACE with RFA group $v$ s. the TACE alone group. The results showed that 2-year survival rate was higher in the TACE with RFA group than in the TACE alone group $\left[\mathrm{RR}=1.675,95 \% \mathrm{CI}: 1.233-2.275, P=0.001, I^{2}=0 \%\right.$ [ Figure 6].

\section{Incidence of fever}

Three studies ${ }^{[11,12,20]}$ (involving 194 participants) compared the incidence of fever of the TACE with RFA group $v s$. the TACE alone group, and showed that there was no significant difference between the two groups $\left[\mathrm{RR}=1.177,95 \% \mathrm{CI}: 0.904-1.532, P=0.227, I^{2}=0 \%\right]$ [Figure 7 ].

\section{Publication bias assessment}

Based on statistical analysis, the meta-analysis of TACE with RFA vs. TACE alone obtained better symmetry of the funnel plot ${ }^{[22]}$ and can be assessed without significant publication bias in the study literature [Figure 8].

\section{DISCUSSION}

Compared to treatment with TACE alone, this study showed that TACE combined with RFA showed significantly better outcomes on tumor response rate $[\mathrm{RR}=1.452,95 \% \mathrm{CI}$ : 1.308-1.610, $P<0.001]$, six-month 


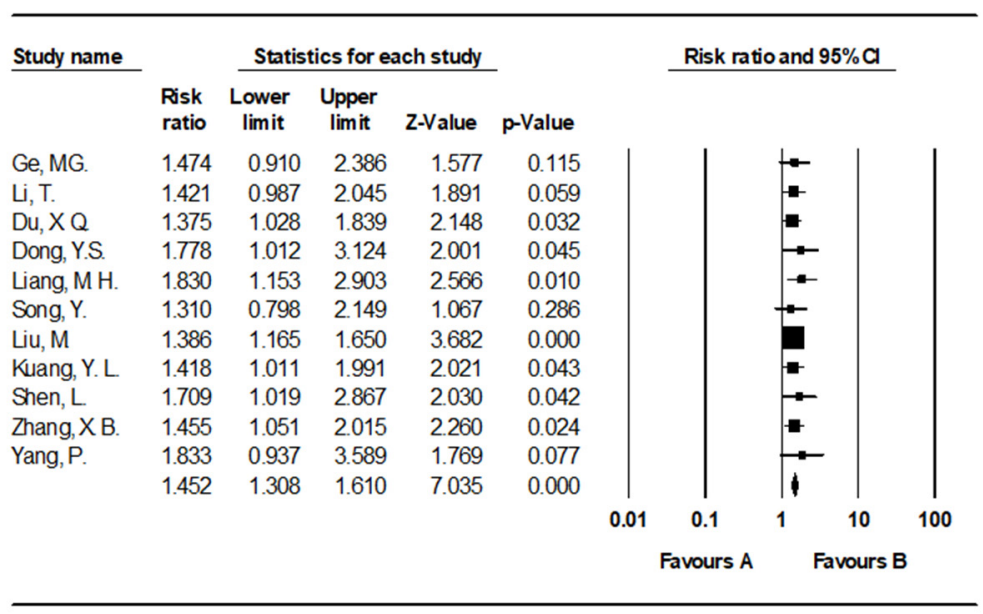

Figure 2. Tumor response rate of comparison TACE with RFA vs. TACE alone



Figure 3. Six-month survival rate of TACE with RFA vs. TACE alone

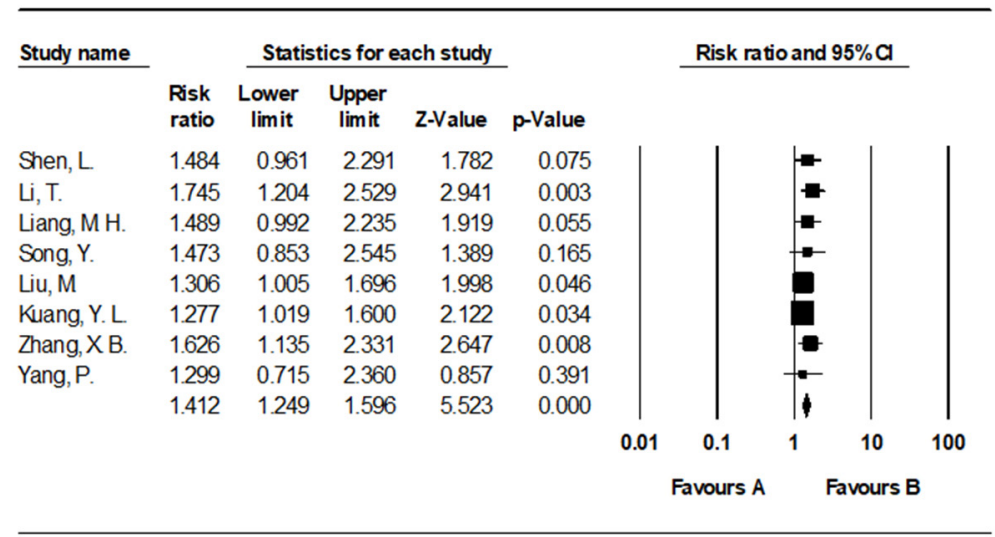

Figure 4. One-year survival rate of TACE with RFA vs. TACE alone

survival rate $[\mathrm{RR}=1.257,95 \% \mathrm{CI}: 1.128-1.401, P<0.001], 1$-year overall survival rate $[\mathrm{RR}=1.412,95 \% \mathrm{CI}$ : 1.249-1.596, $P<0.001]$, eighteen-month survival rate $[\mathrm{RR}=1.792,95 \% \mathrm{CI}: 1.423-2.256, P<0.001]$, and 2 -year overall survival rate $[\mathrm{RR}=1.675,95 \% \mathrm{CI}: 1.233-2.275, P=0.001]$. To our knowledge this study is the first meta-analysis to disclose the efficacy of TACE combined with RFA for HCC tumors larger than $5 \mathrm{~cm}$, compared with TACE alone. The publication bias of this study was evaluated using the symmetry level of the funnel $\mathrm{plot}^{[22]}$. In the analysis of the tumor response rate and survival rate, the symmetry of the shape of the 


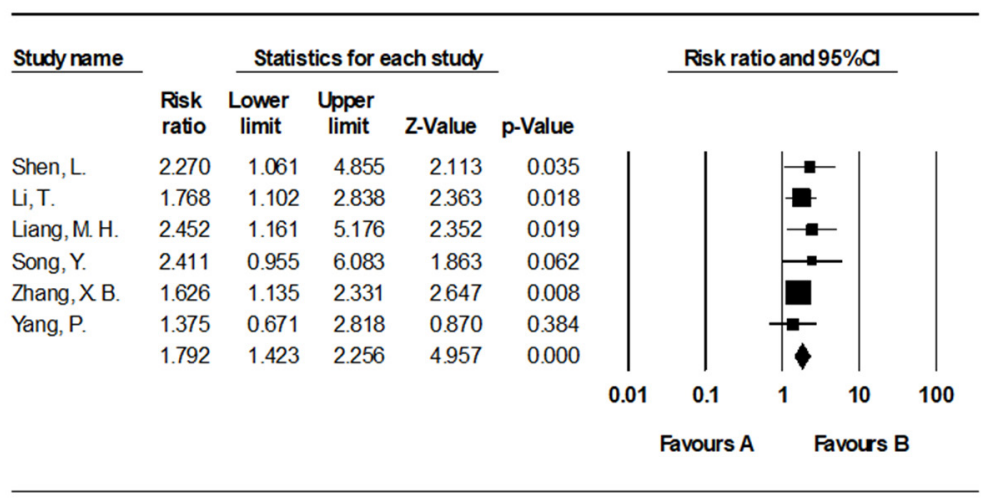

Figure 5. Eighteen-month survival rate of TACE with RFA vs. TACE alone

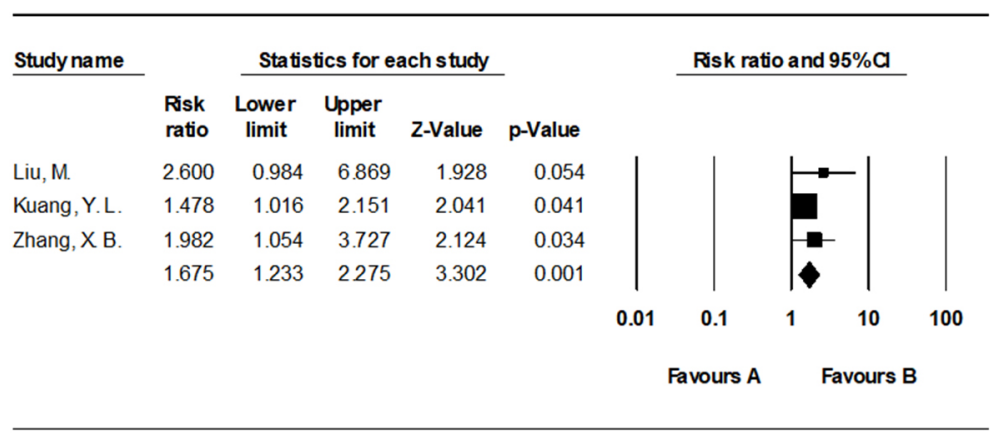

Figure 6. Two-year survival rate of TACE with RFA vs. TACE alone

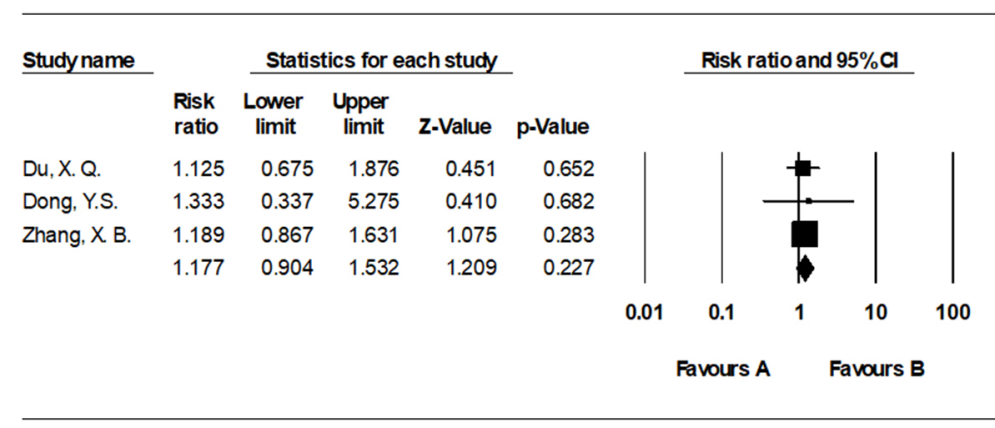

Figure 7. Incidence of fever in the TACE with RFA group vs. TACE alone group

funnel plots indicates that there is no significant bias in this meta-analysis. The overall quality of the studies included in this meta-analysis was evaluated to be of high quality, which gives confidence to our results.

HCC is a serious global health problem and the third most common cause of cancer death. Most patients with HCC are diagnosed with intermediate or advanced stage, with baseline liver dysfunction, intrahepatic metastasis or excessive load, and are not suitable for surgical resection. The established local treatment options include TACE, RFA, ethanol injection, and microwave coagulation; however, it is still unclear which method is the most efficacious ${ }^{[23-25]}$. In the 2018 NCCN Clinical Practice Guidelines for Malignancies, TACE is recommended as a first-line palliative treatment for unresectable HCC. However, the tumor response rate and survival rate of patients treated with TACE alone are not ideal. Therefore, the treatment of TACE combined with other local treatment options such as RFA for comprehensive treatment is gradually being adopted. 
Funnel Plot of Standard Error by Log risk ratio

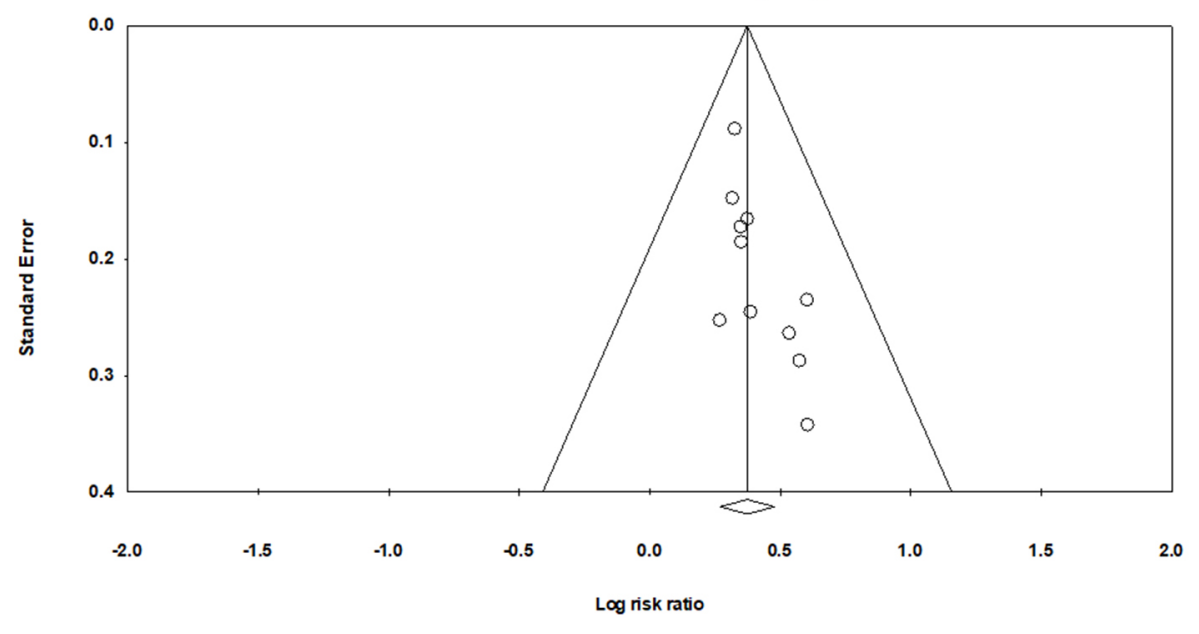

Figure 8. Meta-analysis of funnel plots of tumor response rate

Based on our meta-analysis, combination therapy of TACE with RFA is an effective method for HCC treatment. HCC is mainly supplied by the hepatic artery. Even when the hepatic artery blood flow is blocked by TACE, the thermal coagulation effect of RFA is not affected. Thus, it increases the area of necrosis induced by RFA. Additionally, the effects of expanded ablation zones and anticancer agents on liver cancer cells during treatment may reduce the chance of tumor recurrence ${ }^{[26]}$.

This meta-analysis has some limitations. Firstly, the complications and adverse reactions of combination therapy cannot be assessed fully due to the lack of original research data. Therefore, future studies can further evaluate these indicators. Secondly, the sample size of this current meta-analysis is limited; largescale randomized controlled trials of long-term follow-up are needed to validate this result.

In conclusion, our study suggests that TACE combined with RFA is superior to TACE alone in the treatment of HCC larger than $5 \mathrm{~cm}$. Patients in the combined treatment group showed significantly increased tumor response rate and survival rates compared with those treated with TACE alone. This article provided clinical and systematic evidence for the improved treatment of HCC larger than $5 \mathrm{~cm}$.

\section{DECLARATIONS}

\section{Authors' contributions}

Design of the work: Yang Y, Long Y, Zhang WL

Acquisition, analysis of data: Yang Y, Lv ZM, Yan M

Wrote this paper: Yang Y, Zhang HX, Long Y, Zhang WL

Revised the manuscripts: All authors

\section{Availability of data and materials}

All data did by the authors listed in this paper.

\section{Financial support and sponsorship}

This study was funded by National Natural Science Foundations of China (81773488 and 8177110260).

\section{Conflicts of interest}

All authors declared that there are no conflicts of interest. 


\section{Ethical approval and consent to participate}

Not applicable.

\section{Consent for publication}

Not applicable.

\section{Copyright}

(c) The Author(s) 2019.

\section{REFERENCES}

1. Ferenci P, Fried M, Labrecque D, Bruix J, Sherman M, et al. Hepatocellular carcinoma (HCC): a global perspective. J Clin Gastroenterol 2010;44:239-45.

2. Ferlay J, Shin HR,Bray F, Forman D, Mathers C, et al. Estimates of worldwide burden of cancer in 2008: GLOBOCAN 2008. Int J Cancer 2010;127:2893-917.

3. Yuen MF, Chan AO, Wong BC, Hui CK, Ooi GC, et al. Transarterial chemoembolization for inoperable, early stage hepatocellular carcinoma in patients with Child-Pugh grade A and B: results of a comparative study in 96 Chinese patients. Am J Gastroenterol 2003;98:1181-5

4. Llovet JM, Bruix J. Systematic review of randomized trials for unresectable hepatocellular carcinoma: Chemoembolization improves survival. Hepatology 2003;37:429-42.

5. Marelli L, Shusang V, Buscombe JR, Cholongitas E, Stigliano R, et al. Transarterial injection of (131)I-lipiodol, compared with chemoembolization, in the treatment of unresectable hepatocellular cancer. J Nucl Med 2009;50:871-7.

6. Kim KM, Kim JH, Park IS, Ko GY, Yoon HK, et al. Reappraisal of repeated transarterial chemoembolization in the treatment of hepatocellular carcinoma with portal vein invasion. J Gastroenterol Hepatol 2009;24:806-14.

7. Peng ZW, Zhang YJ, Liang HH, Lin XJ, Guo RP, et al. Recurrent hepatocellular carcinoma treated with sequential transcatheter arterial chemoembolization and RF ablation versus RF ablation alone: a prospective randomized trial. Radiology 2012;262:689-700.

8. Zhao M, Wang JP, Li W, Huang ZL, Zhang FJ, et al. Comparison of safety and efficacy for transcatheter arterial chemoembolization alone and plus radiofrequency ablation in the treatment of single branch portal vein tumor thrombus of hepatocellular carcinoma and their prognosis factors. Zhonghua Yi Xue Za Zhi 2011;91:1167-72.

9. Kim JW, Kim JH, Won HJ, Shin YM, Yoon HK, et al. Hepatocellular carcinomas 2-3 cm in diameter: transarterialchemoembolization plus radiofrequency ablation vs. radiofrequency ablation alone. Eur J Radiol 2012;81:189-93.

10. Shibata T, Isoda H, Hirokawa Y, Arizono S, Shimada K, et al. Small hepatocellular carcinoma: is radiofrequency ablation combined with transcatheter arterial chemoembolization more effective than radiofrequency ablation alone for treatment? Radiology 2009;252:905-13.

11. Dong YS, Zhang ZY, Yang XG. Clinical efficacy of transcatheter arterial chemoembolization combined with radiofrequency ablation for large hepatocellular carcinoma. Chinese Journal of General Surgery 2013;16:486-88.

12. Du XQ. Analysis of the efficacy of transcatheter arterial chemoembolization combined with radiofrequency ablation in the treatment of primary large liver cancer. Clinical Medical Research and Practice 2017;2:15-16.

13. Ge MG, Zhang W. TACE and RFA sequential treatment of 88 cases of advanced liver cancer. Chinese Medical Innovation 2009;6:29.

14. Kuang YL, Wang Z, Yang ZL, Tan ZM. Analysis of short-term efficacy of transcatheter arterial chemoembolization combined with radiofrequency ablation for advanced primary liver cancer. Journal of Hepatobiliary and Pancreatic Surgery 2017;29:363-7.

15. Li T, Jia KD, Zhang KQ. Therapeutic effect of transcatheter arterial chemoembolization combined with ultrasound-guided radiofrequency ablation for large hepatocellular carcinoma. Anhui Medicine 2013;17:1709-11.

16. Liang MH. Hepatic arterial chemoembolization combined with multipolar radiofrequency ablation for the treatment of large liver cancer. Chinese Journal of Gerontology 2011;31:2862-64.

17. Liu M, Yang SF, Wang HL, Gu P, Huang WK. Analysis of the efficacy and prognosis of transcatheter arterial chemoembolization (TACE) combined with percutaneous radiofrequency ablation (RFA) in the treatment of advanced hepatocellular carcinoma. Journal of Practical Cancer 2015.

18. Shen L, Chen MH, Yan K, Yang W, Gao W. To investigate the clinical application of percutaneous radiofrequency ablation combined with hepatic arterial chemoembolization in the treatment of large hepatocellular carcinoma. Chinese Journal of Ultrasound Imaging 2004.

19. Song Y, Jiang SF, Long HY, Zhang YQ. Clinical study of hepatic arterial chemoembolization combined with percutaneous radiofrequency ablation for the treatment of large hepatocellular carcinoma. Advances in Modern and General Surgery 2008;11:203-7.

20. Zhang XB, Wang X, Zheng YH, Zhang XJ. Clinical study of sequential hepatic arterial chemoembolization combined with radiofrequency ablation for large hepatocellular carcinoma. Chinese Journal of Physicians 2016.

21. Yang P, Liang M, Zhang Y, Shen B. Clinical application of a combination therapy of lentinan, multi-electrode RFA and TACE in HCC. Adv Ther 2008;25:787-94.

22. Higgins JP, Altman DG, Gøtzsche PC, Jüni P, Moher D, et al. The Cochrane Collaboration's tool for assessing risk of bias in randomised trials. BMJ 2011;343:d5928.

23. Peng ZW, Zhang YJ, Chen MS, Xu L, Liang HH, et al. Radiofrequency ablation with or without transcatheter arterial chemoembolization in the treatment of hepatocellular carcinoma: a prospective randomized trial. J Clin Oncol 2013;31:426-32. 
24. Cho YK, Kim JK, Kim MY, Rhim H, Han JK. Systematic review of randomized trials for hepatocellular carcinoma treated with percutaneous ablation therapies. Hepatology 2009;49:453-9.

25. Germani G, Pleguezuelo M, Gurusamy K, Meyer T, Isgrò G, et al. Clinical outcomes of radiofrequency ablation, percutaneous alcohol and acetic acid injection for hepatocelullar carcinoma: a meta-analysis. J Hepatol 2010;52:380-8.

26. Peng ZW, Zhang YJ, Liang HH, Lin XJ, Guo RP, et al. Recurrent hepatocellular carcinoma treated with sequential transcatheter arterial chemoembolization and RF ablation versus RF ablation alone: a prospective randomized trial. Radiology 2012;262:689-700. 